\title{
Crystallographic Parameters in Geometrically and Topologically Close-Packed Superstructures
}

\author{
Evgeny A. Knestaypin ${ }^{1, \text { a) }}$, Maksim M. Morozov ${ }^{1, \text { b) }}$, Alexandr I. Potekaev ${ }^{2, c)}$, \\ Anatoly A. Klopotov ${ }^{1,3, d)}$, Tatyana N. Markova, e), and Vladimir D. Klopotov ${ }^{5}$, f) \\ ${ }^{1}$ Tomsk State University of Architecture and Building, Tomsk, 634003, Russia \\ ${ }^{2}$ Siberian Physical-Technical Institute, Tomsk, 634050, Russia \\ ${ }^{3}$ National Research Tomsk State University, Tomsk, 634050, Russia \\ ${ }^{4}$ Siberian State Industrial University, Novokuznetsk, 654007, Russia \\ ${ }^{5}$ National Research Tomsk Polytechnic University, Tomsk, 634050, Russia \\ a) Corresponding author: knestya@gmail.com \\ b)7mmm81@gmal.com \\ c) potekaev@spti.tsu.ru \\ d) klopotovaa@tsuab.ru \\ e) patriotrrf@mail.ru \\ f) vklopotov@mail2000.ru
}

\begin{abstract}
The structures of stoichiometric compositions $\mathrm{AB}, \mathrm{A}_{2} \mathrm{~B}$, and $\mathrm{A}_{3} \mathrm{~B}$ for structures, $\mathrm{B} 19, \mathrm{~L} 1_{0}, \mathrm{~L} 1_{2}, \mathrm{D} 0_{19}, \mathrm{D} 0_{22}$, $\mathrm{D}_{23}, \mathrm{D} 0_{24}, \mathrm{~A} 15, \mathrm{C} 14, \mathrm{C} 15$ and $\mathrm{C} 36$ have been investigated based on the analysis of diagrams in coordinates of spacefilling coefficients $\psi$ on superstructural compression $\Delta \Omega / \Omega$. On the basis of the analysis of the abovementioned diagrams, the equation $\psi=f_{0}+f_{1}(\Delta \Omega / \Omega)$ has been obtained, and coefficients $f_{0}$ and $f_{1}$ of the equation for the investigated structures have been determined. It has been established that values of coefficients $f_{0}$ and $f_{1}$ for Laves phases have higher values than for all other compounds.
\end{abstract}

Keywords: space-filling coefficient, Zen law, intermetallic compounds, superstructural compression

\section{INTRODUCTION}

A wide range of modern industrial alloys include intermetallic compounds (IC), which are included therein as components and determine both mechanical and physical properties. Theoretical and practical interest to IC is caused, primarily, by conditions under which they are stable. In topologically and geometrically close-packed IC the role of various factors (crystallogeometric, thermodynamic, etc.) and various hierarchical levels is different for their formation and their stability. Therefore, the works on searching the common regularities and peculiarities of phase stability in topologically and geometrically close-packed IC affected by crystallogeometric parameters are relevant.

In the literature, the analysis of different crystalline structures is often carried out by allocating similar structural fragments in them. This allowed the authors of the work [1] to generalize the transformation methods that contribute to formation of one or another structural type. A part of the general scheme is shown in Fig. 1. For 67 structural types the structural ancestors are $\mathrm{Mg}$ and $\mathrm{Cu}$. The principle of this systematization is based on the fact that similar positions of certain atoms remain in structural types, which are derived from initial ones by replacing the part of atoms with pairs or groups of atoms. Structural types obtained from the initial ones by multiple substitution may be characterized by the different distribution of certain substituent atoms and groups of substituting atoms [1].

\section{RESEARCH RESULTS}

Structural types, built from similar segments and arranged relative to each other differently, are called homotypic [2]. A special case of homotypic structures are homothetic structures in which layers of the hexagonal symmetry are imposed by the type of closest packing. For example, in the following Laves phases: $\mathrm{MgZn}_{2}-\mathrm{MgCu}_{2}-\mathrm{MgNi}_{2}$ (Figs. 1 and 2). 


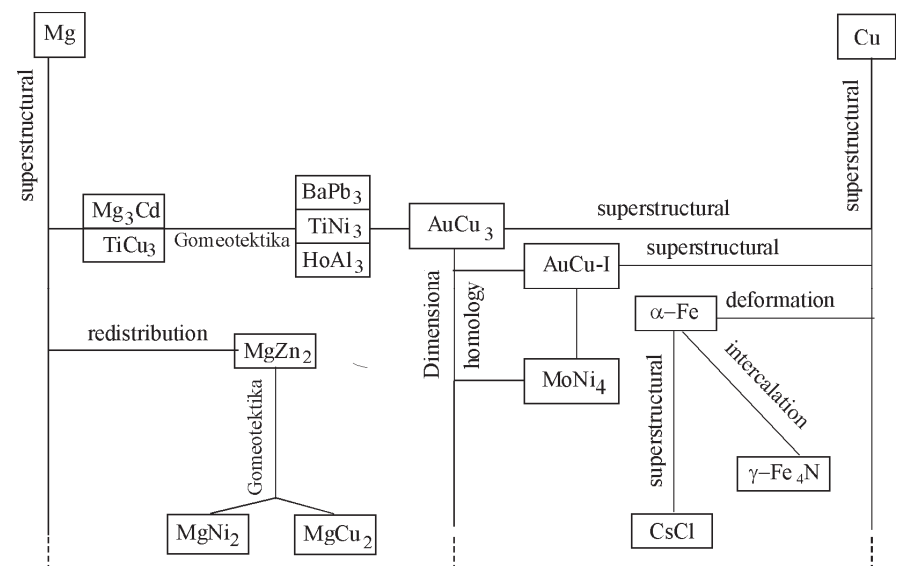

FIGURE 1. A fragment of the diagram of types of affinity, binding together the structural types derived from the closest packing of atoms [1]

Structural types may have similar position of atoms, but different number of components replacing those positions. At the same time, the symmetry and the outlines of the elementary cell may change (Fig. 2). An orderly replacement of regular point systems by various atoms leads to the formation of superstructures. A superstructure can be formed with the constant number of components due to their redistribution to correct point systems. At the same time, during the formation of superstructures, the space groups continue to persist (for example in $\mathrm{Mg}, \mathrm{Mg}_{3} \mathrm{Cd}$ $\left(P 6_{3} / m m c-P 6_{3} / m m c\right)$ ) or do not (in $\mathrm{Cu}, \mathrm{AuCu}(F m 3 m-C 4 / m m m)$ ).

The aim of the work is to carry out a study on peculiarities in the behavior of two integral crystallogeometric parameters: the superstructural compression $(\Delta \Omega / \Omega)$ and the packing coefficient $\psi$ for compounds of two structural types that have two different ancestors: $\mathrm{Mg}\left(\mathrm{Pb}_{3} / \mathrm{mmc}\right)$ and $\mathrm{Cu}(\mathrm{Pm} 3 \mathrm{~m})$. Furthermore, the work is to identify the relationship between the superstructural compression and the space-filling coefficient in intermetallic compounds of the following composition: $\mathrm{AB}, \mathrm{A}_{2} \mathrm{~B}$ and $\mathrm{A}_{3} \mathrm{~B}$. The calculation method and the methodology of the use of these parameters for the analysis of crystalline structures are detailed in [3].

In the work, the space-filling coefficient was calculated by the Laves-Parte method [4]. This coefficient represents the ratio of the volume of atoms contained in the elementary cell (representing hard balls with a known radius) to the volume of the elementary cell $\mathrm{V}$, obtained from the experimental data

$$
\psi=4 / 3 \pi \sum_{i, j}\left(n R_{i}^{3}+m R_{j}^{3}+\ldots\right) V^{-1}
$$

where $n, m, \ldots$ is the number of atoms of the type $i, j, \ldots$ and $R_{i}, R_{j}, \ldots$ are the radii of atoms in the elementary cell of the volume $V$. The relative value of the superstructural compression has been determined using the formula

$$
\begin{aligned}
& \frac{\Delta \Omega}{\Omega}=\frac{\Omega-\Omega^{\text {Zen }}}{\Omega}=1-\frac{\Omega^{\text {Zen }}}{\Omega}, \\
& \Omega^{\text {Zen }}=\Omega_{\mathrm{A}} \cdot \Omega_{\mathrm{A}}+\Omega_{\mathrm{B}} \cdot \Omega_{\mathrm{B}},
\end{aligned}
$$

where $\Omega$ is the atomic volume determined from the experimental data based on the calculation of atomic diameters of elements, calculated from shortest distances in structures; $\Omega^{\mathrm{Zen}}$ is none other than the atomic volume satisfying the Zen law, and determined as the average atomic volume from the expression (3), where $C_{\mathrm{A}}$ and $C_{\mathrm{B}}$ is the concentration of atoms of the type $\mathrm{A}$ and $\mathrm{B}, \Omega_{\mathrm{A}}$ and $\Omega_{\mathrm{B}}$ are atomic volumes of atoms of the type $\mathrm{A}$ and $\mathrm{B}$, respectively. [5]

In our study, Laves phases of the composition $\mathrm{A}_{2} \mathrm{~B}$ were selected as topologically close-packed compounds. These phases belong to the class of Frank-Kasperovskiy phases, possessing topologically close-packed structures. In general, they have the composition $\mathrm{AB}_{2}$ with a large-sized atom in the center of 16-atomic Frank-Kasperovskiy polyhedron, and atoms of a smaller size $B$ in the center of the icosahedron. The group of such ICs includes Laves phases of the composition $\mathrm{AB}_{2}\left(\mathrm{MgCu}_{2}(\mathrm{C} 15), \mathrm{MnZn}_{2}(\mathrm{C} 14)\right.$ and $\left.\mathrm{MgNi}_{2}(\mathrm{C} 36)\right)$.

The IC of the composition $A_{3} B$ based on the FCC lattice with the structure $\mathrm{L}_{2}$ and based on the HCP lattice with structures $\mathrm{D}_{19}$ и $\mathrm{D}_{24}$ have been investigated as geometrically close-packed ordered compounds. Structures $\mathrm{L1}_{0}$, $\mathrm{D}_{22}$ и $\mathrm{D} 0_{23}$ have been investigated based on GCP lattices. 
On the basis of structural data presented in [6], the diagrams in coordinates "packing coefficient $\psi$-superstructural compression $(\Delta \Omega / \Omega)$ " and for structures $\mathrm{A} 15, \mathrm{~B} 2, \mathrm{~B} 19, \mathrm{~L} 1_{0}, \mathrm{L1}_{2}, \mathrm{D} 0_{19}, \mathrm{D} 0_{22}, \mathrm{D} 0_{23}, \mathrm{D} 0_{24}, \mathrm{C} 14$, C15 and C36 (Figs. 3, 4) have been built. The correlation between the space-filling density and the value of superstructural compression has been found. A common pattern has been revealed on a large data array: the greater is the value of superstructural compression, the greater is the space-filling density (Figs. 3, 4).

It has been established that the IC with the $\mathrm{L}_{2}$ structure features a high space-filling coefficient with an average value of about 0.79 . This value exceeds the packing coefficient of 0.74 for pure metals with FCC lattice. But alloys with Laves phases $\mathrm{C} 14$ have a higher $\psi$. The average value of the packaging coefficient for alloys with Laves phases C14 equals to approximately 0.9 . It has been shown that the most numerous group includes alloys with a linear dependence between space-filling coefficients and the superstructure compression.

The search for regularities based on the analysis of dependencies of the space-filling coefficient on the value of the superstructure compression has allowed to divide all considered intermetallic compounds into three groups. The first group includes compounds with a unique dependence between filling-space coefficients and the superstructure compression, and which data can be approximated in the form of a linear dependence:

$$
\psi=f_{0}+f_{1}\left(\frac{\Delta \Omega}{\Omega}\right)
$$

where the first term $f_{0}$ characterizes the value of the space-filling coefficient in compounds when the value of the superstructure contraction equals to zero, and the second term $f_{1}$ is the coefficient of proportionality between $\psi$ and $\Delta \Omega / \Omega$. The numerical values of parameters of the given function are shown in Table 1 .

The second group of intermetallic compounds includes those compounds, in which the data presented on diagrams $\psi$ и $\Delta \Omega / \Omega$ has a large scatter and do not subject to any dependencies. All this raises the question, what is the reason, and what causes a characteristic behavior and the wide scatter of data. Probably, for the first group of alloys a close-packing principle is implemented during the formation of structures [4] and for these compounds it is possible to use the representation of atoms in the shape of "hard spheres" when describing their properties.

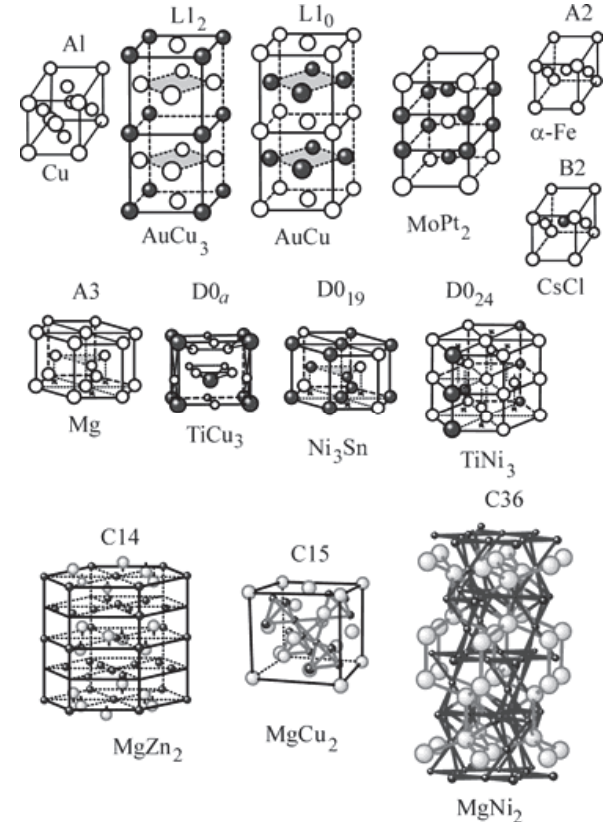

FIGURE 2. Elementary cells of pure metals and compounds
In alloys of the second group such data scatter on diagrams (Figs. 3 and 4) may be conditioned by different reasons. Here are the main ones. First of all, in these structures it is impossible to use the representation of atoms in the shape of "hard spheres". Secondly, the sizes of atoms significantly vary depending on the alloy composition, and their tabular values obtained from the structural data for pure metals cannot be used for the calculation. Thirdly, the structure formation becomes strongly dependent on long-range interatomic interactions. The third group includes those compounds with packing coefficient of alloys $\psi>1$, which has no physical meaning, and is not considered in this study.

The results of structural data processing taken from [6] for intermetallides with non-cubic syngonies with the composition $\mathrm{AB}$ (tetragonal $\mathrm{L1}_{0}$ and orthorhombic B19) are presented in Fig. 3. It is seen that the functional dependency $\psi=f(\Delta \Omega / \Omega)$ retains its linear character, but has different values of parameters (Table 1), and the main array of values for these structures is higher than that of intermetallides with the B2 structure. This indicates a greater packing density in structures with non-cubic syngonies than in structures with cubic syngonies, and suggests that in intermetallides with structures $\mathrm{B} 19$ and $\mathrm{L}_{0}$ the principle of closest packing and the principle of formation of the greatest number of connections begin to play more significant role than in alloys with a BCC lattice, against to another principle, the principle of highest syngony. Most significantly, this phenomenon manifests itself in alloys with Laves phases (Fig. 4). 

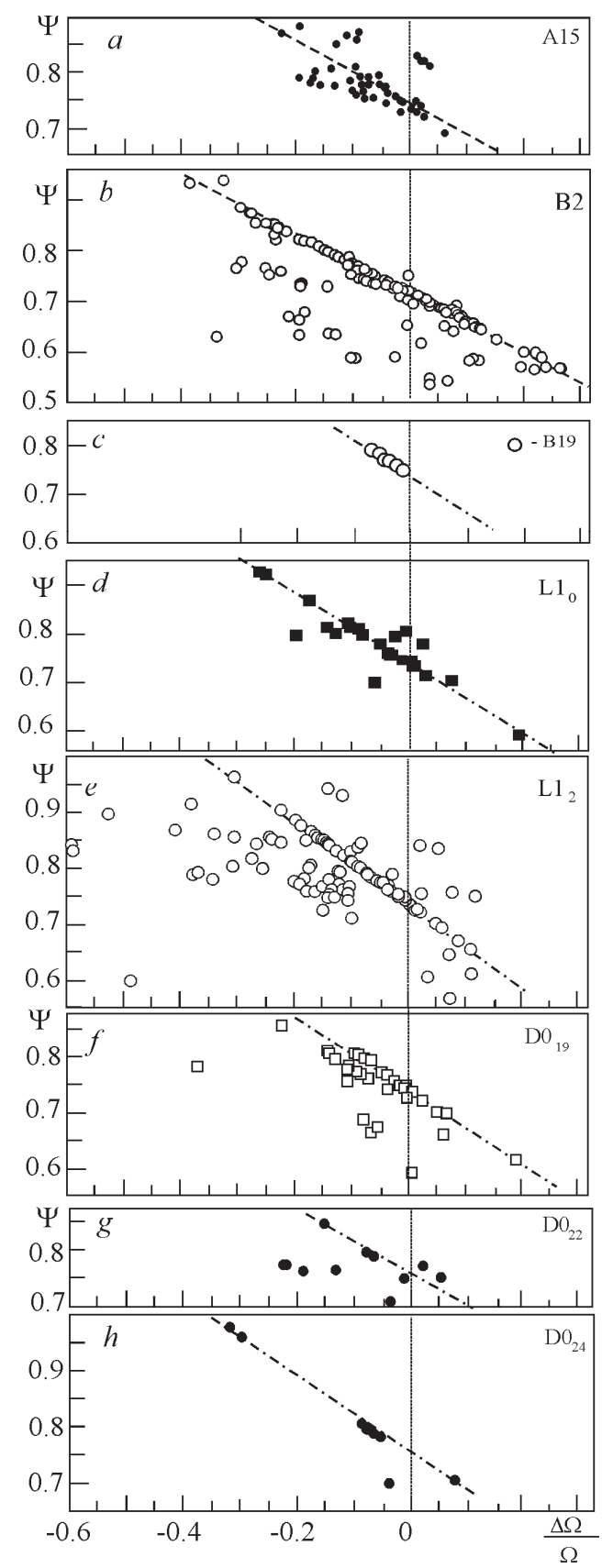

FIGURE 3. The dependence of the packing density coefficient on the superstructural compression in structures:

(a) $\mathrm{A} 15$, (b) B2, (c) B19, (d) L10, (e) L12, (f) D019,

(g) D022, (h) D024. The dashed line corresponds to the equation (4)

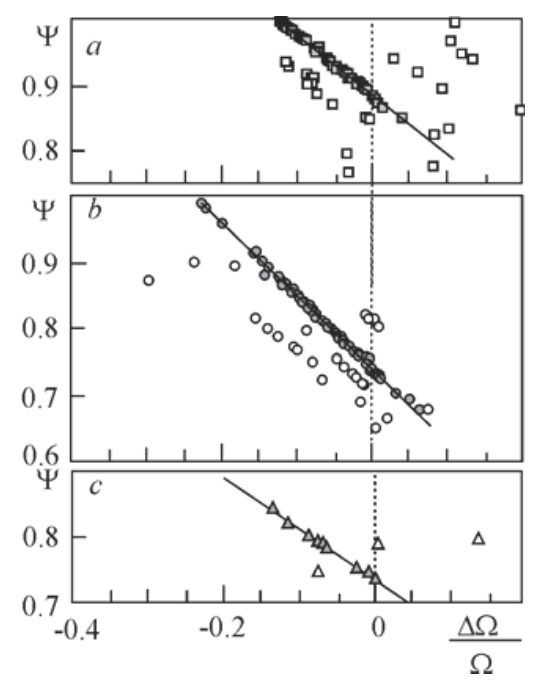

FIGURE 4. The dependence of the packing density coefficient on the superstructural compression in Laves phases: (a) C14,

(b) $\mathrm{C} 15$, (c) C36. The equations following the law (4) are shaded in grey color

Similar diagrams have been obtained for intermetallic compounds in alloys with compositions $A_{3} B$ (Fig. 3). These structures also have large scatter of points, but even among them it is possible to see a set of connections that follow the linear dependence and can be described using the expression (4). The analysis of diagrams and the results of calculations (Table 1) show that intermetallides of the composition $\mathrm{A}_{3} \mathrm{~B}$, on the one hand, reveal two groups of compounds with the coefficient of proportionality $f_{1}$ in the expression (4), which is smaller than in alloys with non-cubic syngonies, and this differs them from intermetallides of the composition $\mathrm{AB}$. On the other hand, the main data array of intermetallides of the composition $\mathrm{A}_{3} \mathrm{~B}$ with a non-cubic syngony is not located higher than points for intermetallides with a cubic structure $\mathrm{L}_{2}$ in $\psi-\Delta \Omega / \Omega$ plots (Fig. 3). This suggests that in intermetallides of the composition $\mathrm{A}_{3} \mathrm{~B}$ the principle of the highest syngony does not reduce its role in formation of intermetallides with the $\mathrm{L}_{2}$ structure.

The analysis of table values of the equation (4) coefficients shows that the majority of compounds have similar values of coefficients $f_{0}$ and $f_{1}$. Alloys with the Laves phase characterized by higher coefficients $f_{0}$ and $f_{1}$ of the equation (4) are out of this group. The ancestor of Laves phases is Mg structure (Fig. 1). Laves phases are formed from the $\mathrm{Mg}$ structure as a result of redistribution of atoms along the allocated $Z$ axis in the form of plane and corrugated grid. 
TABLE 1. Crystallographic data on structures and values of coefficients from the formula (1)

\begin{tabular}{|c|c|c|c|c|c|}
\hline \multirow[t]{2}{*}{ Composition } & \multirow[t]{2}{*}{ Prototype } & \multirow{2}{*}{$\begin{array}{l}\text { Structure } \\
\text { Type }\end{array}$} & \multirow[t]{2}{*}{ Space Group } & \multicolumn{2}{|c|}{$\begin{array}{c}\text { Coefficients from } \\
\text { Formula (4) }\end{array}$} \\
\hline & & & & $f_{0}$ & $f_{1}$ \\
\hline \multirow{3}{*}{$\mathrm{AB}$} & $\mathrm{CsCl}$ & $\mathrm{B} 2$ & $P m 3 m$ & 0.72 & -0.55 \\
\hline & $\mathrm{AuCd}$ & B19 & $P m \mathrm{~cm}$ & 0.74 & -0.80 \\
\hline & $\mathrm{AuCu}$ & $\mathrm{L}_{0}$ & $\mathrm{C} 4 / \mathrm{mmm}$ & 0.74 & -0.67 \\
\hline \multirow{5}{*}{$\mathrm{A}_{3} \mathrm{~B}$} & $\mathrm{AuCu}_{3}$ & $\mathrm{~L}_{2}$ & $P m 3 m$ & 0.74 & -0.73 \\
\hline & $\mathrm{Mg}_{3} \mathrm{Cd}$ & $\mathrm{D} 0_{19}$ & $P 6_{3} / \mathrm{mmm}$ & 0.74 & -0.73 \\
\hline & $\mathrm{Al}_{3} \mathrm{Ti}$ & $\mathrm{D}_{22}$ & $I 4 / \mathrm{mmm}$ & 0.74 & -0.67 \\
\hline & $\mathrm{Al}_{3} \mathrm{Zr}$ & $\mathrm{D} 0_{23}$ & $I 4 / \mathrm{mmm}$ & 0.74 & -0.67 \\
\hline & $\mathrm{Ni}_{3} \mathrm{Ti}$ & $\mathrm{D} 0_{24}$ & $P 6_{3} / \mathrm{mmm}$ & 0.74 & -0.67 \\
\hline \multirow{4}{*}{$\mathrm{A}_{2} \mathrm{~B}$} & $\mathrm{Cr}_{2} \mathrm{Si}$ & A15 & $R m 3 n$ & 0.74 & -0.73 \\
\hline & $\mathrm{MgZn}_{3}$ & $\mathrm{C} 14$ & $P 6_{3} / m m c$ & 0.89 & -0.85 \\
\hline & $\mathrm{MgCu}_{3}$ & $\mathrm{C} 15$ & $F d 3 m$ & 0.74 & -1.08 \\
\hline & $\mathrm{MgNi}_{3}$ & $\mathrm{C} 36$ & $P 6_{3} / m m c$ & 0.74 & -0.76 \\
\hline
\end{tabular}

When grids are mutually placed, the $\mathrm{Mg}$ atom acquires a coordination number 16 . All $\mathrm{Zn}$ atoms have a coordination number 12 and an icosahedral coordination [2].

On the basis of the analysis of dependencies of the space filling coefficient on the value of the superstructural compression of intermetallic compounds of compositions $\mathrm{AB}, \mathrm{A}_{2} \mathrm{~B}$ и $\mathrm{A}_{3} \mathrm{~B}$, two groups of compounds have been allocated. The first group of compounds includes compounds with a linear dependence between the filling-space coefficient and the superstructural compression. The second group of intermetallic compounds includes those, corresponding to data, presented on $\psi-\Delta \Omega / \Omega$ diagrams, and having larger statistical dispersion.

\section{ACKNOWLEDGEMENT}

The authors gratefully acknowledge the support for this research provided by the Tomsk State University Competitiveness Improvement Program.

\section{REFERENCES}

1. E. I. Gladyshevskiy and O. I. Bodak, Crystal Chemistry of Intermetallic Compounds of Rare Earth Metals (Visha Schola, Lviv, 1982).

2. P. I. Krypyakevich, Structural Types of Intermetallic Compounds (Nauka, Moscow, 1977).

3. E. V. Kozlov, A. A. Klopotov, N. O. Solonitsina, and A. S. Tailashev, Russ. Phys. J. 49, 35 (2006).

4. W. Pearson, The Crystal Chemistry and Physics of Metals and Alloys (Wiley-Interscience, London, 1972).

5. E. Zen, J. Mineralogist Soc. America 41, 523 (1934).

6. N. P. Lyakishev, State Diagrams of Binary Metallic Systems (Mechanical Engineering, Moscow, 1996-2000), V. 1-3. 